\title{
Effect of Nitrogen Fertilizer and Fungicide Application on Disease Severity, Yield and Yield Related Traits of Emmer Wheat (Triticum diccocom L.) in Highlands of Bale, Southeastern Ethiopia
}

\author{
Wubishet Alemu*, Tilahun Bayisa \\ Oromia Agricultural Research Institute, Sinana Agricultural Research Center, Bale-Robe, Ethiopia \\ Email address: \\ wubtesema@gmail.com (W. Alemu), tilahunbayisa@gmail.com (T. Bayisa) \\ ${ }^{*}$ Corresponding author
}

\section{To cite this article:}

Wubishet Alemu, Tilahun Bayisa. Effect of Nitrogen Fertilizer and Fungicide Application on Disease Severity, Yield and Yield Related Traits of Emmer Wheat (Triticum diccocom L.) in Highlands of Bale, Southeastern Ethiopia. Plant. Vol. 4, No. 2, 2016, pp. 8-13.

doi: $10.11648 /$ j.plant.20160402.11

Received: June 14, 2016; Accepted: June 25, 2016; Published: August 6, 2016

\begin{abstract}
Emmer wheat is produced mostly in the highlands of Ethiopia, including Bale. There is a significant amount of emmer wheat in Bale zone during both bona (main) and gena (short) cropping seasons and is one of the major crops in the region. However, its production and productivity is curtailed by leaf rust which is caused by Puccinia tritici and nitrogen fertilizer management. A field experiment was conducted to determine the effect of nitrogen fertilizer on emmer wheat production and to evaluate integrated effect of nitrogen fertilizer and fungicide application on disease severity, yield and yield related traits of emmer wheat at two locations from 2012-2014 main cropping season. Treatments were composed of one improved variety (Sinana-01), fungicide spray timing: at growth stages of (GS) 33-36, 33-36\&59-69, nil and complete control (at 7 days interval) and nitrogen fertilizer: at the rate of 0,23, 46 and $69 \mathrm{~kg} / \mathrm{ha}$. Plots were set in factorial arrangement laid in randomized complete block design in three replications. There was difference among the four levels of $\mathrm{N}$-fertilizer application in grain yield and leaf rust severity. The highest and lowest grain yield was obtained from $0-23 \mathrm{~kg} / \mathrm{ha}$ and $46-69 \mathrm{~kg} / \mathrm{ha} \mathrm{N}$ fertilizer, respectively. Maximum mean disease severity was recorded from maximum $\mathrm{N}$-fertilizer application. Difference was observed among fungicide treatments in controlling leaf rust. Highest mean leaf rust severity was recorded on nil fungicide application while the lowest from fungicide application at 7-days interval (complete control). Optimum level of nitrogen fertilizer $(0-23 \mathrm{~kg} / \mathrm{ha})$ and at least one time spray of fungicide should be recommended for emmer wheat production in Bale.
\end{abstract}

Keywords: Emmer Wheat, Fungicide, Leaf Rust, N-fertilizer, Puccinia tritici f.sp. tritici, Severity, Triticum Diccocom

\section{Introduction}

A complex of several subspecies represents the tetraploid group of wheat (Triticum spp.). The most important cultivated types are Emmer (Triticum diccocom) and durum wheat (Triticum durum). The most likely progenitor of emmer is Triticum dicoccoides, which in turn, may be a chromosome-hybrid of $T$. boeoticum and a species of Aegilops (Helbaeck, 1970).

Emmer wheat was domesticated in the Near East somewhere in the Fertile Crescent. It is speculated that early immigrants of Hamites introduced wheat to Ethiopia highlands, 5,000 years ago and emmer wheat was the first to be introduced (Helbaeck, 1970; Feldman, 1979).

Agriculture in Ethiopia is still predominantly traditional thus the tetraploid wheats grown by farmers are landraces, consisting of a mixture of genetic lines, which vary, in botanical form and morphological characters. Emmer wheat has been a minor crop in most parts of Ethiopian highlands but is being replaced by tetraploid and hexploid wheat, which free threshing unlike emmer wheat. Today, emmer wheat is grown on a limited scale and comprises about 7 percent of Ethiopia's entire wheat production (BOSTID, 1996). Emmer wheat is produced mostly in the highlands of Ethiopia, 
including Bale, Arsi, Shewa, Harerge, Wello, Gojam and Gondar. There is a significant amount of emmer in Bale zone during meher (June to September) and belg (March to May) production seasons and it is one of the major crops in the region and contributes more than T. durum (Demissie and Hailegiorgis, 1985). Similarly, the 5 years (1991-1995) data of Bale Zone Bureau of Agriculture indicate emmer wheat ranking fifth in acreage after bread wheat, barley, maize and tef. However, the bread wheat based extension program coupled with the problem of thresh ability in emmer wheat has been resulting in the decline of emmer production in the southeast highlands of Ethiopia.

In Ethiopia emmer wheat is known by different local name as Aja in Amharic, Hyssa or Matajebo in Oromo language and Arras in Tigrigna. It is also used in various ways as food in the farming community. Some are ground into flour and baked into unfermented bread kita (pancake). Some are crushed and cooked with milk or water to make soft porridge; split grains of emmer are also mixed with boiling water and butter to produce hard porridge. Moreover, emmer is used to prepare cankita (local spaghetti) in Bale highland (Phillips, 1995; Tesfaye, 2000).

Farmers in most areas of the country assert that they produce emmer wheat for its special medicinal and nutritional values. Traditionally it is believed that broken bones heal faster when emmer is consumed in the form of porridge. As a result it is recommended for mothers as special diet in maintaining their health and strength after childbirth. Generally, the local people appreciate emmer as compared to other wheat in that emmer does not affect stomach when consumed in any form and considered as healthy food (Tesemma and Belay, 1991; Tesfaye, 2002).

Emmer wheat has the ability to grow in poor soil condition and marginal land. It is drought tolerant and resistant to a range of fungal diseases except leaf rust to which emmer is susceptible (Damania, et al., 1992; BOSTID, 1996; Nesbitt and Samuel, 1996). In addition other economically useful traits such as disease resistance, earliness and frost tolerance can be exploited for wheat improvement (ICARDA, 1991; Beteselassieet al., 2007; Tekluet al., 2007). But its production and productivity is declining through time due to various reasons. Among these, diseases and inappropriate nitrogen fertilizer management are the major ones. So this project is initiated to determine the effect of nitrogen fertilizer on emmer wheat production and to evaluate integrated effect of nitrogen fertilizer and fungicide application on disease severity, yield and yield related traits of emmer wheat.

\section{Materials and Methods}

The experiment was conducted in factorial arrangement laid in randomized complete block design in three replications at two locations, Sinana on-farm (Selka) and Goba (Aloshe) during 2012-14 main cropping seasons. It was planted in a plot size of $4 \mathrm{mx} 5 \mathrm{~m}$ and the distance between plots and blocks was $1 \mathrm{~m}$ and $1.5 \mathrm{~m}$, respectively. Treatments were composed of one improved variety (Sinana-01), four fungicide spray schedules: GS (growth stage of the crop) 33$36,33-36 \& 59-69$, nil and complete control (at 7 days interval starting from onset of the disease) and four levels of nitrogen fertilizer: 0, 23, 46 and $69 \mathrm{~kg} / \mathrm{ha}$. Seeds were planted in broadcast. Recommended seed rate $100 \mathrm{~kg} / \mathrm{ha}$ and fertilizer di ammonium phosphate (DAP) $100 \mathrm{~kg} / \mathrm{ha}$ was used at planting except plots with 0 level nitrogen. Triple super phosphate (TSP) was used for plots with 0 level of nitrogen fertilizer. Land preparation and weeding culture was done as recommended for wheat. Fungicide (Tilt 250 EC) was applied at the rate of $0.5 \mathrm{l} / \mathrm{ha}$ mixing with $250 \mathrm{l} / \mathrm{ha}$ water using knapsack sprayer. Disease severity was recorded in percentage by using modified Cob-scale (Peterson et al., 1948) on plot bases of each treatment. Agronomic data: number of tillers $/ \mathrm{m}^{2}$, spike length, number of spike $/ \mathrm{m}^{2}$, seed/spike, plant height, days to maturity, biomass yield and grain yield were collected.

Statistical Analyses

Analysis of Variance (ANOVA) was done by using SAS GLM Procedure (SAS version 9.00, Inst. 2002) and means comparisons for the significantly different variables were made among treatments using Least Significant Differences (LSD) test at 0.05 level of significance.

\section{Results and Discussion}

Statistical analysis of the data showed that there was no significant difference among the four levels of $\mathrm{N}$-fertilizer application in their impact on tillers $/ \mathrm{m}^{2}$, spike length, number of spike $/ \mathrm{m}^{2}$, seed/spike, plant height, days to maturity, biomass yield and disease severity at Selka. But there was significant difference in grain yield between 0 and $69 \mathrm{~kg} / \mathrm{ha}$ $\mathrm{N}$-fertilizer (Table 1). Even though there was no significant difference among the four levels of $\mathrm{N}$-fertilizer, the highest grain yield $(3775.50 \mathrm{~kg} / \mathrm{ha})$, tiller $/ \mathrm{m}^{2}(207.00)$ and $\mathrm{spike} / \mathrm{m}^{2}$ (245.83) was obtained from $0 \mathrm{~kg} / \mathrm{ha} \mathrm{N}$-fertilizer application. Contrary, the highest leaf rust severity (18.08\%) was recorded from $69 \mathrm{~kg} / \mathrm{ha} \mathrm{N}$-fertilizer application while the lowest $(12.58 \%)$ from $0 \mathrm{~kg} / \mathrm{ha} \mathrm{N}$-fertilizer application in spite of insignificant difference among the different rates of $\mathrm{N}$ fertilizer application on disease severity. It was also observed that there was no significant difference among the four levels of fungicide on tillers $/ \mathrm{m}^{2}$, number of $\mathrm{spike} / \mathrm{m}^{2}$, seed $/ \mathrm{spike}$, plant height, days to maturity, biomass yield and grain yield at Selka (Sinana on-farm). However, there was significant difference between nil and 7-days interval application of fungicide on spike length and leaf rust severity (Table 2). The highest $(36.50 \%)$ and the lowest $(6.58 \%)$ leaf rust severity were recorded on nil and 7-days interval fungicide application, respectively. Conversely, the highest and the lowest spike length was recorded on 7-days interval and nil fungicide application, respectively.

At Goba almost similar result was obtained for the treatments. Likely, there was no significant difference among the four levels of $\mathrm{N}$-fertilizer application on tillers $/ \mathrm{m}^{2}$, spike length, seed/spike, days to maturity and leaf rust severity. A significant difference was observed between 0 and 46 and 69 
$\mathrm{kg} / \mathrm{ha} \mathrm{N}$-fertilizer application on plant height. Similarly, there was significant difference in grain yield for 0 and $23 \mathrm{~kg} / \mathrm{ha}$ $\mathrm{N}$-fertilizer application. The highest $(2934.30 \mathrm{~kg} / \mathrm{ha})$ grain yield was also obtained from $23 \mathrm{~kg} /$ ha $\mathrm{N}$-fertilizer application (Table 3). Relatively, maximum disease severity was recorded from $46 \mathrm{~kg} / \mathrm{ha} \mathrm{N}$-fertilizer application.

As it is witnessed from table, there was no significant difference among the four fungicide application frequencies on tillers $/ \mathrm{m}^{2}$, seed/spike, plant height, days to maturity and grain yield at Goba (Table 4). However, there was significant difference between nil and GS 33-36fungicide application in spike length. Similarly, there was significant difference between nil and GS 33-36 \& 59-69 and 7-days interval fungicide application in disease severity. Likely, relatively maximum disease severity was obtained from nil application while the minimum from GS33-36 \& 59-69 and 7-days interval fungicide application.

Table 1. Effect of nitrogen fertilizer rate on disease severity, yield, and yield related traits of improved emmer wheat variety (Sinana-01) at Selka in 2013 main cropping season.

\begin{tabular}{|c|c|c|c|c|c|c|c|c|c|}
\hline $\mathrm{N}$-fertilizer levels & Tiller $/ \mathbf{m}^{2}$ & SL (cm) & Spike/m² & Seed/Spike & PH (cm) & DM & LR. Severity (\%) & $\begin{array}{l}\text { Biomass } \\
\text { (ton/ha) }\end{array}$ & $\begin{array}{l}\text { Grain yield } \\
\text { (kg/ha) }\end{array}$ \\
\hline $0 \mathrm{~kg} / \mathrm{ha} \mathrm{N}$ & $207.00^{\mathrm{a}}$ & $6.50^{\mathrm{a}}$ & $245.83^{\mathrm{a}}$ & $19.00^{\mathrm{a}}$ & $123.33^{\mathrm{a}}$ & $144.92^{\mathrm{a}}$ & $12.58^{\mathrm{a}}$ & $11.46^{\mathrm{a}}$ & $3775.50^{\mathrm{a}}$ \\
\hline $46 \mathrm{~kg} / \mathrm{ha} \mathrm{N}$ & $201.00^{\mathrm{a}}$ & $7.25^{\mathrm{a}}$ & $242.33^{\mathrm{a}}$ & $20.17^{\mathrm{a}}$ & $126.25^{\mathrm{a}}$ & $145.92^{\mathrm{a}}$ & $17.25^{\mathrm{a}}$ & $12.64^{\mathrm{a}}$ & $3262.10^{\mathrm{ab}}$ \\
\hline $69 \mathrm{~kg} / \mathrm{ha} \mathrm{N}$ & $199.00^{\mathrm{a}}$ & $7.58^{\mathrm{a}}$ & $240.67^{\mathrm{a}}$ & $19.75^{\mathrm{a}}$ & $127.17^{\mathrm{a}}$ & $146.17^{\mathrm{a}}$ & $18.08^{\mathrm{a}}$ & $12.47^{\mathrm{a}}$ & $3016.50^{\mathrm{b}}$ \\
\hline Mean & 202.71 & 7.02 & 242.17 & 19.85 & 124.94 & 145.92 & 15.81 & 12.04 & 3407.90 \\
\hline $\operatorname{LSD}(0.05)$ & 38.75 & 1.23 & 45.78 & 1.79 & 4.37 & 2.62 & 5.67 & 1.66 & 568.43 \\
\hline
\end{tabular}

$\mathrm{SL}=$ Spike Length, $\mathrm{PH}=$ Plant Height, $\mathrm{DM}=$ Days to maturity, $\mathrm{LR}=$ Leaf Rust

Table 2. Effect of fungicide frequency on disease severity, yield, and yield related traits of improved emmer wheat variety (Sinana-01) at Selka in 2013 main cropping season.

\begin{tabular}{|c|c|c|c|c|c|c|c|c|c|}
\hline Fungicide schedules & Tiller $/ \mathbf{m}^{2}$ & SL (cm) & Spike/m² & Seed/Spike & PH (cm) & DM & $\begin{array}{l}\text { LR. Severity } \\
(\%)\end{array}$ & $\begin{array}{l}\text { Biomass } \\
\text { (ton/ha) }\end{array}$ & $\begin{array}{l}\text { Grain yield } \\
\text { (kg/ha) }\end{array}$ \\
\hline Nil application & $201.17^{\mathrm{a}}$ & $6.25^{\mathrm{b}}$ & $239.83^{\mathrm{a}}$ & $19.67^{\mathrm{a}}$ & $124.25^{\mathrm{a}}$ & $144.42^{\mathrm{a}}$ & $36.50^{\mathrm{a}}$ & $12.01^{\mathrm{a}}$ & $3524.20^{\mathrm{a}}$ \\
\hline Fungicide application at (GS33-36) & $208.33^{\mathrm{a}}$ & $6.92^{\mathrm{ab}}$ & $248.67^{\mathrm{a}}$ & $20.33^{\mathrm{a}}$ & $123.83^{\mathrm{a}}$ & $146.67^{\mathrm{a}}$ & $11.58^{\mathrm{b}}$ & $12.06^{\mathrm{a}}$ & $3521.90^{\mathrm{a}}$ \\
\hline Fungicide application at (GS33-36 \& 59-69) & $193.67^{\mathrm{a}}$ & $7.42^{\mathrm{ab}}$ & $234.33^{\mathrm{a}}$ & $20.42^{\mathrm{a}}$ & $126.67^{\mathrm{a}}$ & $146.58^{\mathrm{a}}$ & $8.58^{\mathrm{b}}$ & $11.94^{\mathrm{a}}$ & $3247.70^{\mathrm{a}}$ \\
\hline 7-days interval application & $207.67^{\mathrm{a}}$ & $7.50^{\mathrm{a}}$ & $245.83^{\mathrm{a}}$ & $19.00^{\mathrm{a}}$ & $125.00^{\mathrm{a}}$ & $146.00^{\mathrm{a}}$ & $6.58^{\mathrm{b}}$ & $12.15^{\mathrm{a}}$ & $3337.80^{\mathrm{a}}$ \\
\hline Mean & 202.71 & 7.02 & 242.17 & 19.85 & 124.94 & 145.92 & 15.81 & 12.04 & 3407.90 \\
\hline $\mathrm{CV}(\%)$ & 22.99 & 21.07 & 22.73 & 10.86 & 4.15 & 2.56 & 43.15 & 16.57 & 20.06 \\
\hline
\end{tabular}

$\mathrm{SL}=$ Spike Length, $\mathrm{PH}=$ Plant Height, DM=Days to maturity, $\mathrm{LR}=$ Leaf Rust

Table 3. Effect of nitrogen fertilizer rate on disease severity, yield, and yield related traits of improved emmer wheat variety (Sinana-01) at Goba (Aloshe) in 2013 main cropping season.

\begin{tabular}{|c|c|c|c|c|c|c|c|}
\hline N-fertilizer levels & Tiller $/ \mathbf{m}^{2}$ & SL (cm) & Seed/Spike & PH (cm) & DM & LR. Severity (\%) & $\begin{array}{l}\text { Grain yield } \\
\text { (kg/ha) }\end{array}$ \\
\hline $0 \mathrm{~kg} / \mathrm{ha} \mathrm{N}$ & $126.75^{\mathrm{a}}$ & $6.90^{\mathrm{a}}$ & $38.25^{\mathrm{a}}$ & $86.25^{b}$ & $135.00^{\mathrm{a}}$ & $0.57^{\mathrm{a}}$ & $2138.10^{\mathrm{b}}$ \\
\hline $23 \mathrm{~kg} / \mathrm{ha} \mathrm{N}$ & $148.50^{\mathrm{a}}$ & $6.78^{\mathrm{a}}$ & $38.40^{\mathrm{a}}$ & $89.25^{\mathrm{ab}}$ & $133.67^{\mathrm{a}}$ & $0.80^{\mathrm{a}}$ & $2934.30^{\mathrm{a}}$ \\
\hline $46 \mathrm{~kg} / \mathrm{ha} \mathrm{N}$ & $146.42^{\mathrm{a}}$ & $6.80^{\mathrm{a}}$ & $38.87^{\mathrm{a}}$ & $93.92^{\mathrm{a}}$ & $134.08^{\mathrm{a}}$ & $1.23^{\mathrm{a}}$ & $2877.70^{\mathrm{ab}}$ \\
\hline $69 \mathrm{~kg} / \mathrm{ha} \mathrm{N}$ & $149.00^{\mathrm{a}}$ & $6.94^{\mathrm{a}}$ & $37.35^{\mathrm{a}}$ & $93.08^{\mathrm{a}}$ & $142.50^{\mathrm{a}}$ & $1.00^{\mathrm{a}}$ & $2794.40^{\mathrm{ab}}$ \\
\hline Mean & 142.67 & 6.86 & 6.87 & 90.63 & 136.31 & 0.90 & 2686.129 \\
\hline CV (\%) & 24.39 & 9.23 & 38.22 & 7.76 & 10.62 & - & 33.45 \\
\hline
\end{tabular}

$\mathrm{SL}=$ Spike Length, $\mathrm{PH}=$ Plant Height, $\mathrm{DM}=$ Days to maturity, $\mathrm{LR}=$ Leaf Rust

Table 4. Effect of fungicide frequency on disease severity, yield, and yield related traits of improved emmer wheat variety (Sinana-01) at Goba (Aloshe) in 2013 main cropping season.

\begin{tabular}{|c|c|c|c|c|c|c|c|}
\hline Fungicide schedules & Tiller $/ \mathbf{m}^{2}$ & SL (cm) & Seed/Spike & PH (cm) & DM & $\begin{array}{l}\text { LR. Severity } \\
(\%)\end{array}$ & $\begin{array}{l}\text { Grain } \\
\text { yield (kg/ha) }\end{array}$ \\
\hline Nil application & $150.58^{\mathrm{a}}$ & $7.14^{\mathrm{a}}$ & $37.82^{\mathrm{a}}$ & $91.58^{\mathrm{a}}$ & $134.00^{\mathrm{a}}$ & $1.53^{\mathrm{a}}$ & $3036.00^{\mathrm{a}}$ \\
\hline Fungicide application at (GS33-36) & $141.67^{\mathrm{a}}$ & $6.61^{\mathrm{b}}$ & $37.90^{\mathrm{a}}$ & $90.83^{\mathrm{a}}$ & $134.00^{\mathrm{a}}$ & $0.87^{\mathrm{ab}}$ & $2613.40^{\mathrm{a}}$ \\
\hline Fungicide application at (GS33-36 \& 59-69) & $130.17^{\mathrm{a}}$ & $6.92^{\mathrm{ab}}$ & $38.00^{\mathrm{a}}$ & $90.00^{\mathrm{a}}$ & $142.75^{\mathrm{a}}$ & $0.60^{\mathrm{b}}$ & $2635.10^{\mathrm{a}}$ \\
\hline 7-days interval application & $148.25^{\mathrm{a}}$ & $6.76^{\mathrm{ab}}$ & $39.15^{\mathrm{a}}$ & $90.08^{\mathrm{a}}$ & $134.50^{\mathrm{a}}$ & $0.60^{\mathrm{b}}$ & $2459.90^{\mathrm{a}}$ \\
\hline Mean & 142.67 & 6.86 & 6.87 & 90.63 & 136.31 & 0.90 & 2686.129 \\
\hline CV (\%) & 24.39 & 9.23 & 38.22 & 7.76 & 10.62 & - & 33.45 \\
\hline
\end{tabular}

$\mathrm{SL}=$ Spike Length, $\mathrm{PH}=$ Plant Height, $\mathrm{DM}=$ Days to maturity, $\mathrm{LR}=$ Leaf Rust 
In 2014 cropping season, statistical analysis of the data showed that there was no significant difference among the four levels of $\mathrm{N}$-fertilizer application in their impact on spike length, number of spike $/ \mathrm{m}^{2}$, seed/spike, seed/spikelet, plant height, days to maturity, biomass yield and disease (leaf rust) severity at Selka. But there was significant difference in grain yield between 0 and 23 and $69 \mathrm{~kg} / \mathrm{ha} \mathrm{N}$ fertilizer (Table 5). Even though there was no significant difference among the four levels of $\mathrm{N}$-fertilizer, the highest grain yield $(5031.00 \mathrm{~kg} / \mathrm{ha})$ was obtained from $0 \mathrm{~kg} / \mathrm{ha} \mathrm{N}$ fertilizer application. Similarly, the highest leaf rust severity (11.03\%) was recorded from $0 \mathrm{~kg} / \mathrm{ha} \mathrm{N}$-fertilizer application while the lowest $(8.72 \%)$ from $46 \mathrm{~kg} / \mathrm{ha} \mathrm{N}$-fertilizer application in spite of insignificant difference among the different rates of $\mathrm{N}$-fertilizer application on disease severity. This result is contrary to the last years result on the effect of $\mathrm{N}$-fertilizer rate for the control of leaf rust disease. It was also observed that there was no significant difference among the four levels of fungicide application on spike length, number of spike/ $\mathrm{m}^{2}$, seed/spike, seed/spikelet, plant height, days to maturity and biomass yield at Selka (Sinana on-farm). However, there was significant difference among fungicide application schedules on leaf rust severity (Table 6 ). The highest $(12.11 \%)$ and the lowest $(7.08 \%)$ leaf rust severity was recorded on nil and 7-days interval fungicide application, respectively. Conversely, the highest and the lowest grain yield was recorded on 7-days interval and nil fungicide application, respectively. It is witnessed from table 2 is that there was significant difference in grain yield among 7-days interval fungicide application and the rest of fungicide treatments.

Significant difference was observed among the four levels of $\mathrm{N}$-fertilizer application in spike $/ \mathrm{m}^{2}$, seed/spike, biomass yield, grain yield and leaf rust severity at Goba (Table 7). The highest $(5779.10 \mathrm{~kg} / \mathrm{ha})$ grain yield was obtained from $0 \mathrm{~kg} / \mathrm{ha} \mathrm{N}$-fertilizer application while the lowest from $46 \mathrm{~kg} / \mathrm{ha} \mathrm{N}$-application. Relatively, maximum mean disease severity $(8.61 \%)$ was recorded from $46 \mathrm{~kg} / \mathrm{ha} \mathrm{N}$-fertilizer application while the lowest on $0 \mathrm{~kg} / \mathrm{ha} \mathrm{N}$-fertilizer. This result clearly shows that the lowest grain yield was obtained from the highly diseased plot.

As it is witnessed from table 4, there was no significant difference among the four fungicide application treatments on spike length, spike $/ \mathrm{m}^{2}$, seed/spike, seed/spikelet, plant height, biomass yield and grain yield at Goba (Table 8). In spite of insignificant difference among the four levels of fungicide application, relatively the highest grain yield (5431.30 $\mathrm{kg} / \mathrm{ha}$ ) was obtained from complete control (fungicide application at seven days interval). There was significant difference among the fungicide application treatments in controlling leaf rust disease. However, there was no significant difference between fungicide application at GS33-36 \& 59-69) and seven days interval. The highest mean disease severity $(11.75 \%)$ was recorded on nil fungicide application while the lowest $(5.07 \%)$ from fungicide application at seven days interval.

The results of this study demonstrated that leaf rust disease pressure on emmer wheat varies across locations and over years in Bale. In the present study, the leaf rust disease pressure was higher in 2014 cropping season than 2013 at Goba district, whereas at Sinana district (Selka), it was higher in 2013 than 2014 cropping season. In areas and seasons where the disease pressure is higher, fungicide application at seven days interval (completed control) will provide complete control of the disease severity and give maximum grain yield with acceptable quality. However, fungicide application at seven days interval for total control of the disease is not economical compared to emmer wheat productivity per hectare. The current study clearly showed at least one time fungicide application reduced the leaf rust severity to the lowest level possible and gave maximum grain yield and quality at both locations which is economical for small scale farmers.

As it is witnessed from the results of this study, maximum grain yield was obtained from the lowest nitrogen fertilizer treatment. This is because as the level of nitrogen fertilizer increases emmer wheat is susceptible to lodging, deep green, weak, succulent and susceptible to diseases which in turn results in low grain yield and quality. Moreover, the current study showed that an optimum amount of nitrogen fertilizer, $0-23 \mathrm{~kg} / \mathrm{ha}$ revealed maximum grain yield and lowest disease severity at both locations and during both cropping years.

Table 5. Effect of nitrogen fertilizer rate on disease severity, yield, and yield related traits of improved emmer wheat variety (Sinana-01) at Selka in 2014 main cropping season.

\begin{tabular}{llllllllll}
\hline $\begin{array}{l}\text { N-fertilizer } \\
\text { levels }\end{array}$ & SL (cm) & Spike/m & Seed/Spike & Seed/spikelet & PH (cm) & DM & LR. Severity (\%) & $\begin{array}{l}\text { Biomass } \\
\text { (ton/ha) }\end{array}$ & $\begin{array}{l}\text { Grain yield } \\
(\mathbf{k g} / \mathbf{h a})\end{array}$ \\
\hline $0 \mathrm{~kg} / \mathrm{ha} \mathrm{N}$ & $6.71^{\mathrm{a}}$ & $573.50^{\mathrm{a}}$ & $17.42^{\mathrm{a}}$ & $1.83^{\mathrm{a}}$ & $113.72^{\mathrm{a}}$ & $135.42^{\mathrm{a}}$ & $11.03^{\mathrm{a}}$ & $38.00^{\mathrm{a}}$ & $5031.00^{\mathrm{a}}$ \\
$23 \mathrm{~kg} / \mathrm{ha} \mathrm{N}$ & $6.74^{\mathrm{a}}$ & $642.67^{\mathrm{a}}$ & $18.33^{\mathrm{a}}$ & $1.87^{\mathrm{a}}$ & $114.02^{\mathrm{a}}$ & $134.83^{\mathrm{a}}$ & $9.07^{\mathrm{a}}$ & $37.17^{\mathrm{a}}$ & $3906.20^{\mathrm{b}}$ \\
$46 \mathrm{~kg} / \mathrm{ha} \mathrm{N}$ & $6.94^{\mathrm{a}}$ & $555.42^{\mathrm{a}}$ & $19.43^{\mathrm{a}}$ & $1.93^{\mathrm{a}}$ & $114.20^{\mathrm{a}}$ & $134.83^{\mathrm{a}}$ & $8.72^{\mathrm{a}}$ & $38.33^{\mathrm{a}}$ & $3849.10^{\mathrm{b}}$ \\
$69 \mathrm{~kg} / \mathrm{ha} \mathrm{N}$ & $7.04^{\mathrm{a}}$ & $623.75^{\mathrm{a}}$ & $19.62^{\mathrm{a}}$ & $1.80^{\mathrm{a}}$ & $114.32^{\mathrm{a}}$ & $134.50^{\mathrm{a}}$ & $9.67^{\mathrm{a}}$ & $39.42^{\mathrm{a}}$ & $3924.40^{\mathrm{ab}}$ \\
Mean & 6.86 & 598.83 & 18.70 & 1.86 & 114.06 & 134.90 & 9.62 & 38.23 & 4177.67 \\
CV (\%) & 7.67 & 21.34 & 14.63 & 12.29 & 3.65 & 1.37 & 35.76 & 10.31 & 32.34 \\
LSD (0.05) & 0.43 & 105.50 & 2.26 & 0.19 & 3.44 & 1.52 & 2.84 & 3.26 & 1115.60 \\
\hline
\end{tabular}

$\mathrm{SL}=$ Spike Length, $\mathrm{PH}=$ Plant Height, $\mathrm{DM}=$ Days to maturity, $\mathrm{LR}=$ Leaf Rust 
Table 6. Effect of fungicide frequency on disease severity, yield, and yield related traits of improved emmer wheat variety (Sinana-01) at Selka in 2014 main cropping season.

\begin{tabular}{|c|c|c|c|c|c|c|c|c|c|}
\hline Fungicide schedules & $\begin{array}{l}\text { SL } \\
(\mathrm{cm})\end{array}$ & Spike/m² & Seed/Spike & Seed/spikelet & PH (cm) & DM & $\begin{array}{l}\text { LR. Severity } \\
(\%)\end{array}$ & $\begin{array}{l}\text { Biomass } \\
\text { (ton/ha) }\end{array}$ & $\begin{array}{l}\text { Grain yield } \\
\text { (kg/ha) }\end{array}$ \\
\hline Nil application & $6.94^{\mathrm{a}}$ & $579.58^{\mathrm{a}}$ & $19.23^{\mathrm{a}}$ & $1.90^{\mathrm{a}}$ & $114.12^{\mathrm{a}}$ & $134.42^{\mathrm{a}}$ & $12.11^{\mathrm{a}}$ & $37.83^{\mathrm{a}}$ & $3432.40^{\mathrm{b}}$ \\
\hline Fungicide application at (GS33-36) & $7.00^{\mathrm{a}}$ & $597.67^{\mathrm{a}}$ & $19.10^{\mathrm{a}}$ & $1.85^{\mathrm{a}}$ & $112.70^{\mathrm{a}}$ & $134.92^{\mathrm{a}}$ & $9.13^{\mathrm{bc}}$ & $37.25^{\mathrm{a}}$ & $4426.80^{\mathrm{ab}}$ \\
\hline Fungicide application at (GS33-36 \& 59-69) & $6.82^{\mathrm{a}}$ & $641.50^{\mathrm{a}}$ & $18.30^{\mathrm{a}}$ & $1.77^{\mathrm{a}}$ & $115.92^{\mathrm{a}}$ & $135.17^{\mathrm{a}}$ & $10.16^{\mathrm{ab}}$ & $39.25^{\mathrm{a}}$ & $3849.80^{\mathrm{b}}$ \\
\hline 7-days interval application & $6.68^{\mathrm{a}}$ & $576.58^{\mathrm{a}}$ & $18.17^{\mathrm{a}}$ & $1.92^{\mathrm{a}}$ & $113.52^{\mathrm{a}}$ & $135.08^{\mathrm{a}}$ & $7.08^{c}$ & $38.58^{\mathrm{a}}$ & $5001.70^{\mathrm{a}}$ \\
\hline Mean & 6.86 & 598.83 & 18.70 & 1.86 & 114.06 & 134.90 & 9.62 & 38.23 & 4177.67 \\
\hline $\mathrm{CV}(\%)$ & 7.67 & 21.34 & 14.63 & 12.29 & 3.65 & 1.37 & 35.76 & 10.31 & 32.34 \\
\hline $\operatorname{LSD}(0.05)$ & 0.43 & 105.50 & 2.26 & 0.19 & 3.44 & 1.52 & 2.84 & 3.26 & 1115.60 \\
\hline
\end{tabular}

$\mathrm{SL}=$ Spike Length, $\mathrm{PH}=$ Plant Height, $\mathrm{DM}=$ Days to maturity, $\mathrm{LR}=$ Leaf Rust

Table 7. Effect of nitrogen fertilizer rate on disease severity, yield, and yield related traits of improved emmer wheat variety (Sinana-01) at Goba (Aloshe) in 2014 main cropping season.

\begin{tabular}{|c|c|c|c|c|c|c|c|c|c|}
\hline N-fertilizer levels & SL (cm) & Spike/m² & Seed/Spike & Seed/spikelet & PH (cm) & DM & LR. Severity (\%) & $\begin{array}{l}\text { Biomass } \\
\text { (ton/ha) }\end{array}$ & $\begin{array}{l}\text { Grain yield } \\
\text { (kg/ha) }\end{array}$ \\
\hline $0 \mathrm{~kg} / \mathrm{ha} \mathrm{N}$ & $6.58^{\mathrm{a}}$ & $459.75^{b}$ & $19.50^{\mathrm{b}}$ & $2.11^{\mathrm{a}}$ & $117.10^{\mathrm{a}}$ & $122.83^{\mathrm{a}}$ & $6.01^{\mathrm{b}}$ & $25.54^{\mathrm{b}}$ & $5779.10^{\mathrm{a}}$ \\
\hline $23 \mathrm{~kg} / \mathrm{ha} \mathrm{N}$ & $6.44^{\mathrm{a}}$ & $568.92^{a}$ & $20.03^{\mathrm{ab}}$ & $2.03^{\mathrm{a}}$ & $116.15^{\mathrm{a}}$ & $122.67^{a}$ & $8.25^{\mathrm{ab}}$ & $26.08^{\mathrm{ab}}$ & $5160.10^{\mathrm{ab}}$ \\
\hline $46 \mathrm{~kg} / \mathrm{ha} \mathrm{N}$ & $7.38^{\mathrm{a}}$ & $520.83^{\mathrm{ab}}$ & $19.75^{\mathrm{b}}$ & $2.11^{\mathrm{a}}$ & $117.13^{\mathrm{a}}$ & $121.33^{\mathrm{a}}$ & $8.43^{\mathrm{a}}$ & $29.75^{\mathrm{a}}$ & $5006.80^{\mathrm{ab}}$ \\
\hline $69 \mathrm{~kg} / \mathrm{ha} \mathrm{N}$ & $7.15^{\mathrm{a}}$ & $439.17^{b}$ & $20.78^{\mathrm{a}}$ & $2.08^{\mathrm{a}}$ & $119.30^{\mathrm{a}}$ & $121.75^{\mathrm{a}}$ & $8.61^{\mathrm{a}}$ & $29.50^{\mathrm{a}}$ & $4302.40^{\mathrm{b}}$ \\
\hline Mean & 6.89 & 497.17 & 20.02 & 2.09 & 117.42 & 122.15 & 7.82 & 27.72 & 5062.10 \\
\hline CV (\%) & 9.53 & 22.19 & 6.12 & 8.19 & 5.93 & 1.58 & 36.07 & 16.16 & 26.66 \\
\hline LSD (0.05) & 0.54 & 91.13 & 1.01 & 0.14 & 5.75 & 1.59 & 2.33 & 3.69 & 1114.40 \\
\hline
\end{tabular}

$\mathrm{SL}=$ Spike Length, $\mathrm{PH}=$ Plant Height, $\mathrm{DM}=$ Days to maturity, $\mathrm{LR}=$ Leaf Rust

Table 8. Effect of fungicide frequency on disease severity, yield, and yield related traits of improved emmer wheat variety (Sinana-01) at Goba (Aloshe) in 2014 main cropping season.

\begin{tabular}{|c|c|c|c|c|c|c|c|c|c|}
\hline Fungicide schedules & $\begin{array}{l}\text { SL } \\
(\mathrm{cm})\end{array}$ & Spike/m² & Seed/Spike & Seed/spikelet & PH (cm) & DM & $\begin{array}{l}\text { LR. Severity } \\
(\%)\end{array}$ & $\begin{array}{l}\text { Biomass } \\
\text { (ton/ha) }\end{array}$ & $\begin{array}{l}\text { Grain yield } \\
\text { (kg/ha) }\end{array}$ \\
\hline Nil application & $6.94^{\mathrm{a}}$ & $497.08^{\mathrm{a}}$ & $19.77^{\mathrm{a}}$ & $2.12^{\mathrm{a}}$ & $116.68 \mathrm{a}$ & $120.67^{b}$ & $11.75^{\mathrm{a}}$ & $27.75^{\mathrm{a}}$ & $5026.40^{\mathrm{a}}$ \\
\hline Fungicide application at (GS33-36) & $6.68^{\mathrm{a}}$ & $491.75^{\mathrm{a}}$ & $19.97^{\mathrm{a}}$ & $2.10^{\mathrm{a}}$ & $118.92^{\mathrm{a}}$ & $121.83^{\mathrm{ab}}$ & $8.90^{\mathrm{b}}$ & $27.63^{\mathrm{a}}$ & $5094.90^{\mathrm{a}}$ \\
\hline Fungicide application at (GS33-36 \& 59-69) & $6.74^{\mathrm{a}}$ & $456.58^{\mathrm{a}}$ & $20.13^{\mathrm{a}}$ & $2.03^{\mathrm{a}}$ & $114.65^{\mathrm{a}}$ & $122.67^{\mathrm{a}}$ & $5.57^{\mathrm{c}}$ & $25.92^{\mathrm{a}}$ & $4695.80^{\mathrm{a}}$ \\
\hline 7-days interval application & $7.18^{\mathrm{a}}$ & $543.25^{\mathrm{a}}$ & $20.20^{\mathrm{a}}$ & $2.10^{\mathrm{a}}$ & $119.43^{\mathrm{a}}$ & $123.42^{\mathrm{a}}$ & $5.07^{\mathrm{c}}$ & $29.58^{\mathrm{a}}$ & $5431.30^{\mathrm{a}}$ \\
\hline Mean & 6.89 & 497.17 & 20.02 & 2.09 & 117.42 & 122.15 & 7.82 & 27.72 & 5062.10 \\
\hline CV (\%) & 9.53 & 22.19 & 6.12 & 8.19 & 5.93 & 1.58 & 36.07 & 16.16 & 26.66 \\
\hline LSD $(0.05)$ & 0.54 & 91.13 & 1.01 & 0.14 & 5.75 & 1.59 & 2.33 & 3.69 & 1114.40 \\
\hline
\end{tabular}

$\mathrm{SL}=$ Spike Length, $\mathrm{PH}=$ Plant Height, $\mathrm{DM}=$ Days to maturity, $\mathrm{LR}=$ Leaf Rust

\section{Conclusions}

Generally from the two years data and over locations result it was observed that the highest disease severity was recorded from nil application of fungicide and from the highest $\mathrm{N}$ fertilizer application. An optimum level of $\mathrm{N}$-fertilizer (0-23 $\mathrm{kg} / \mathrm{ha}$ ) application at planting and at least one time spray of fungicide $(0.5 \mathrm{~L} / \mathrm{ha})$ at $5 \%$ severity level should be recommended for emmer wheat production in Bale, southeastern Ethiopia.

\section{Acknowledgments}

The study was funded by Oromia Agricultural Research Institute. Cereal crops research case team members of Sinana Agricultural Research Center contributed technical and professional assistances for the completion of this work.

\section{References}

[1] Beteselassie N, Fininsa C, Badebo A. 2007. Sources of Resistance to Stem Rust (Pucciniagraminis f. sp. tritici) in Ethiopian Tetraploid Wheat Accessions. Genetic Resources and Crop Evolution 54: 337-343.

[2] BOSTID (Board on science and technology for international development, National Research Council (BSTIDNRC) (1996). Lost crops of Africa, Vol. I Grains. The National Academy of science. National academic press, Washington D. C USA.

[3] Damania AB, Hakims and Moualla MY. (1992). Evolution of variation in $T$. dicoccon of wheat improvement in stress environment. Hereditas 116: 163-66.

[4] Demissie A and Hailegiorgis M. (1985). "Belg" collecting in Bale administrative region. PGRC/E-ILCA Germplasm Newsletter 10: 6-11. 
[5] Feldman M. (1979). Wheats (Triticum spp.). Evolution of Crop Plants. Simmonds N. W (ed.) Longman Group Limited, London.

[6] Helback H. (1970). Commentary on the phylogenesis of Triticumand Hordeum. Econ. Bot. 20: 350-60.

[7] ICARDA. (1991). Genetic Resource Unit Annual Report for 1990. Aleppo, Syria: ICARDA.

[8] Nesbitt $M$ and Smuael D. (1996). From staple crop to extinction? The archaeology and history of the hulled wheat. In: Hulled wheats, promoting the conservation and used of underutilized and neglected crops.

[9] Peterson RF, Campbell AB and Hannah AE. 1948. A diagrammatic scale for estimating rust intensity of leaves and stem of cereals. Canadian J. Res. Sect. C. 26: 496-500.

[10] Phillip S. (1995). Poaceae (Gramineae). Flora of Ethiopia and Eritrea. Vol. 7. Hederg. I and Edwards S., (eds.). Addis Ababa, Ethiopia, Uppsala, Sweden.
[11] Teklu Y, Hammer K and Röder MS. 2007. Simple sequence repeats marker polymorphism in emmer wheat (Triticumdicoccon Schrank): Analysis of genetic diversity and differentiation. Genetic Resources and Crop Evolution 54: 543-554.

[12] Tesemma $\mathrm{T}$ and Balay G. (1991). Aspects of Ethiopian tetraploid wheat with emphasis on durum wheat genetics and breeding Research. A historical perspective (Gebremariam, H., Tanner, D. G, and Hulluka, M., (eds.) Addis Ababa, Ethiopia.

[13] Tesfaye K. 2000. Morphological and Biochemical Diversity of Emmer Wheat (Triticumdicoccum) in Ethiopia M.Sc. Thesis Addis Ababa University, Science Faculty, Department of Biology, Applied Genetics Section.

[14] Tesfaye K, Asfaw Z and Bekele E. 2002. Agroethnobotanical Study of Emmer Wheat (Triticumdicoccum) in Southeastern Highlands of Ethiopia. A paper presented at the workshop of $8^{\text {th }}$ International of Congress of International Society of Ethnobiology. 